Proceedings of the International Symposium on Physics of Materials (ISPMA 14), September 10-15, 2017, Prague

\title{
Microstructure, Texture, and Magnetic Properties of GOES
}

\author{
L. KalvodA*, J. ČApeK, K. Kolařík, M. KuČERÁKová and S. VRatislav \\ Czech Technical University in Prague, Faculty of Nuclear Science and Physical Engineering, \\ Břehová 7, 11519 Prague 1, Czech Republic
}

\begin{abstract}
A series of Fe3Si grain oriented electric steel samples are investigated as a function of grain size and the level of perfection of the dominating Goss texture. Characterization of morphology, crystallographic texture and magnetic properties is performed by light microscopy, scanning electron microscopy, electron backscatter diffraction, neutron diffraction and magnetic Barkhausen noise methods. The experiments are conducted on the "as fabricated" specimens and then on the specimen sheets with the protective coating removed. Juxtaposition of the obtained results provides information about the complex links between the texture, microstructure, and the resulting magnetic properties of the specimens observed at different excitation conditions.
\end{abstract}

DOI: 10.12693/APhysPolA.134.824

PACS/topics: 81.05.Bx, 75.50.Bb, 81.70.-q, 75.60.Ej, 75.78.Fg, 75.30.Cr, 75.30.Gw

\section{Introduction}

Grain oriented electric steel (GOES) sheets are indispensable as magnetic flux conductors in construction of high power electric devices such as transformers or electric engines. In spite of the very high perfection level achieved in production technology of the GOES sheets, several percent of the total annual production of electric energy is still dissipated in magnetic cores, making any development leading to further reduction of these losses highly desirable.

The total losses occurring in GOES at the standard ac power conditions (frequency $f=50 \div 60 \mathrm{~Hz}$ ) can be roughly considered as consisting of the following three comparatively large contributions: classical eddy currents, hysteresis losses, and excess eddy currents [1]. Reduction of the frequency independent hysteresis losses (related to the processes of magnetization and demagnetization) is achieved through optimization of the microstructure, namely crystallographic preferential orientation (texture) and the grain size of the GOES sheets. The key step features the fabrication of the so-called Goss texture characterized by the ideal orientation $\{110\}\langle 001\rangle$ in the Euler space of rotations. Influence of the classical eddy currents is suppressed by addition of $c a .3$ mass percent of silicon reducing the specific electric conductivity of GOES, utilization of thin sheets suppressing the bulk eddy currents, and application of electrically resistive surface coatings. The excess eddy currents loss is related to the effects of magnetization non-uniformity in the sheet volume caused e.g. by presence of pinning sites at grain boundaries, impurities, precipitates, and also by the additional tensile stress introduced by protective coating layer, influencing the actual magnetic domain structure. Reduction of the excessive (anomalous)

\footnotetext{
* corresponding author; e-mail ladislav.kalvoda@fji.cvut.cz
}

losses is obtained by careful optimization of the GOES microstructure, especially focused on preparation of materials with extremely large oriented grains.

In the present paper, we provide summary of the results obtained on a series of nine GOES samples covering the commercial material scale from the standard GOES of moderately large grains (several millimeters in diameter), the Goss texture of a medium sharpness and the specific magnetic losses P1.5T well over $1 \mathrm{~W} / \mathrm{kg}$ (P1.5T measured at the magnetic flux level of $1.5 \mathrm{~T}$ ), up to the highly optimized GOES with extremely large grains (several tens of millimeters), very sharp Goss texture and the value of $\mathrm{P} 1.5 \mathrm{~T}$ below $1 \mathrm{~W} / \mathrm{kg}$.

\section{Experimental}

\subsection{Samples}

The GOES samples under study have been supplied in form of standard Epstein sheets with the dimensions $30 \times 300 \mathrm{~mm}^{2}$. The samples are marked as S1-S9, the rising order following the growing value of the saturation magnetic flux $\left(B_{s}\right)$. For further experiments, square specimens with a side length of $30 \mathrm{~mm}$ were cut from the original long strips. The basic characteristics of the samples provided by their producers are summarized in Table I. Standard methods of light microscopy, scanning electron microscopy (SEM) and electron backscatter diffraction (EBSD) were applied in further characterization of morphology and microstructure of the samples.

\subsection{Neutron texture analysis}

The diffraction patterns were collected on the KSN-2 neutron diffractometer situated at one of the horizontal channels of the nuclear research reactor LVR-15 at the Nuclear Research Institute, plc. Rez, Czech Republic. For every tested GOES sample, stack of 6 square sheets was mounted in Huber Eulerian cradle 511.5 goniometer and used in place of the specimen for neutron diffraction experiments. The resulting thickness of specimens varied within $1.2-2 \mathrm{~mm}$. Orientation of crystalline 
Characteristics of the investigated GOES samples S1-S9: thickness $t$, saturation magnetic flux $B_{s}$, and specific power loss $\mathrm{P} 1.5 \mathrm{~T}$ and $\mathrm{P} 1.7 \mathrm{~T}$ measured at power frequency $50 \mathrm{~Hz}$ and magnetic flux level $1.5 \mathrm{~T}$ and $1.7 \mathrm{~T}$, respectively, by standard Epstein frame method.

\begin{tabular}{l|c|c|c|c|c|c|c|c|c}
\hline \hline \multicolumn{1}{c|}{ Sample } & S1 & S2 & S3 & S4 & S5 & S6 & S7 & S8 & S9 \\
\hline$t[\mathrm{~mm}]$ & 0.31 & 0.31 & 0.31 & 0.25 & 0.31 & 0.27 & 0.27 & 0.25 & 0.26 \\
$B_{s}[\mathrm{~T}]$ & 1.646 & 1.663 & 1.703 & 1.749 & 1.763 & 1.826 & 1.834 & 1.872 & 1.894 \\
P1.5T $[\mathrm{W} / \mathrm{kg}]$ & 1.168 & 1.116 & 1.005 & 0.898 & 1.002 & 0.874 & 0.756 & 0.756 & 0.774 \\
P1.7T $[\mathrm{W} / \mathrm{kg}]$ & 1.830 & 1.760 & 1.570 & 1.375 & 1.470 & 1.227 & 1.054 & 1.033 & 1.043
\end{tabular}

grains ( $\alpha$-Fe phase) was described in the Cartesian coordination system (c.c.s.) of specimen defined by the rolling direction (RD), transversal direction (TD), and normal direction (ND) and satisfying the orthorhombic symmetry. Two different experimental procedures were used in characterization of the Goss texture order. The first method (described in detail in [2]) is based on determination of full pole figures (PFs) of $\{110\},\{200\}$ and $\{211\}$ poles followed by numerical calculation of orientation distribution function (ODF) of grains and its integration over a 15-degrees surroundings of the $\{110\}\langle 001\rangle$ ideal orientation in the Euler space of rotations. The procedure provides the relative volume $V_{O D F}$ of those grains that are oriented within the specified section of the Euler space. The second method [3] explores in detail the distribution of $\{100\}$ poles within a 10 degrees' surroundings of RD. Numeric integration of the map obtained for the tested specimen provides value of the parameter $V_{[001]}$ equal to the relative volume of grains with the specified orientation, i.e. characterizing the integral level of fibre texture $\langle 100\rangle \|$ RD.

\subsection{Magnetic measurements}

Magneto-elastic analyser Rollscan 300 (Stresstech Oy, Finland) equipped with the software MicroScan 600-1 and the standard probe S1-138-15-0 was used in measurements of coercive force $H_{c}$, remanence $B_{r}$, relative permeability $\mu_{r}$, and the root mean square (RMS) signal of the magnetic Barkhausen noise (BN). A sinusoidal modulation of the excitation magnetic field $H$ was applied with the frequency $45 \mathrm{~Hz}$ and voltage on the probe either $2.5 \mathrm{~V}$ or $12.5 \mathrm{~V}$, corresponding to the $H$ field intensities $c a .4 .1 \mathrm{kA} / \mathrm{m}$ and $10.0 \mathrm{kA} / \mathrm{m}$ (the values obtained from calibration measurements). The resulting values of the measured parameters were obtained as a statistical average of three subsequent measurements performed on three randomly selected places, the signal at each position recorded over 10 periods of the field. Semicircular azimuthal scan of the excitation yoke orientation was performed with step of 5 angular degrees. Single square sheet of the tested GOES material was used in place of the specimen, all sheets at first tested "as fabricated" and then characterized after removal of the surface coating layer. The electrochemical etching was performed on LectroPol-5 (Struers GmbH) using the standard electrolyte solution A2. Thickness of the removed layer was kept on $20 \mu \mathrm{m}$ for all specimens.

\section{Results and discussion}

The results of the basic texture studies and magnetic measurements performed on samples S1-S9 are summarized in Table II. All the here shown values relate to the properties along RD. There is a clear correlation between the values of saturation flux $B_{s}$ and the texture parameters, $V_{O D F}$ and $V_{[001]}$ for all samples. The Pearson correlation coefficient $r$ amounts 0.950 and 0.828 , respectively. For the correlation coefficient between $B_{s}$ and the power loss $\mathrm{P} 1.5 \mathrm{~T}$ and $\mathrm{P} 1.7 \mathrm{~T}$ we get value $r=-0.949$ and -0.978 , respectively. The highest correlation to $B_{s}$ is found for the loss difference (P1.7T - P1.5T): $\mathrm{r}=0.992$.

The obtained texture and magnetic parameters along RD: the grain volume ratios $V_{[001]}$ and $V_{O D F}(c f$. the

TABLE II text), coercive force $H_{c}$, remanence $B_{r}$, relative permeability $\mu_{r}$ and BN RMS; the magnetic parameters measured at the indicated voltage levels. The Pearson correlation coefficient $(-1 \leq r \leq 1)$ quantifies the probabilistic level of the linear dependence between $B_{s}$ and the parameter on the given row.

\begin{tabular}{l|c|c|c|c|c|c|c|c|c|c}
\hline \hline \multicolumn{1}{c|}{ Sample } & S1 & S2 & S3 & S4 & S5 & S6 & S7 & S8 & S9 & $r$ \\
\hline$V_{\text {[001] }[\%]^{a}}$ & 28.8 & 25.5 & 29.2 & 39.2 & 36.5 & 45 & 39.5 & 34.9 & 49.8 & 0.828 \\
$V_{O D F}[\%]$ & 26.8 & 27.9 & 30.4 & 28.5 & 33.2 & 34.4 & 34.9 & 37.0 & 40.2 & 0.950 \\
$H_{c} 12.5 \mathrm{~V}^{a}[\mathrm{~mA} / \mathrm{m}]$ & 70.1 & 58.1 & 52.8 & 100.9 & 47.4 & 71.5 & 71.5 & 79.5 & 119.5 & 0.549 \\
$H_{c} 2.5 \mathrm{~V}[\mathrm{~mA} / \mathrm{m}]$ & 12.7 & 35.4 & 39.4 & 86.2 & 6.0 & 51.4 & 24.7 & 38.1 & 62.1 & 0.302 \\
$B_{r} 12.5 \mathrm{~V}^{a}[\mathrm{mT}]$ & 342.8 & 178.5 & 193.8 & 589.3 & 243.1 & 639.3 & 405.4 & 1258.5 & 879 & 0.771 \\
$B_{r} 2.5 \mathrm{~V}[\mathrm{mT}]_{\mu_{r} 12.5 \mathrm{~V}^{a}}^{51.1}$ & 82.4 & 95.1 & 261.6 & 25.0 & 157.4 & 80.1 & 92.3 & 241.9 & 0.400 \\
$\mu_{r} 2.5 \mathrm{~V}$ & 5288 & 3277 & 4030 & 6074 & 5349 & 10156 & 6285 & 17177 & 8432 & 0.713 \\
RMS $12.5 \mathrm{~V}^{a}$ & 2721 & 1928 & 2167 & 3178 & 2458 & 3019 & 3292 & 4292 & 2655 & 0.627 \\
RMS U $=2.5 \mathrm{~V}$ & 111.0 & 81.3 & 95.4 & 138.4 & 127.8 & 222.1 & 136.9 & 332.6 & 161.5 & 0.704 \\
\hline$a$ & 83.1 & 72.1 & 74.1 & 116.3 & 98.5 & 115.9 & 124.6 & 69.4 & 135.1 & 0.566 \\
\hline
\end{tabular}

${ }^{a}$ after Ref. [3] 

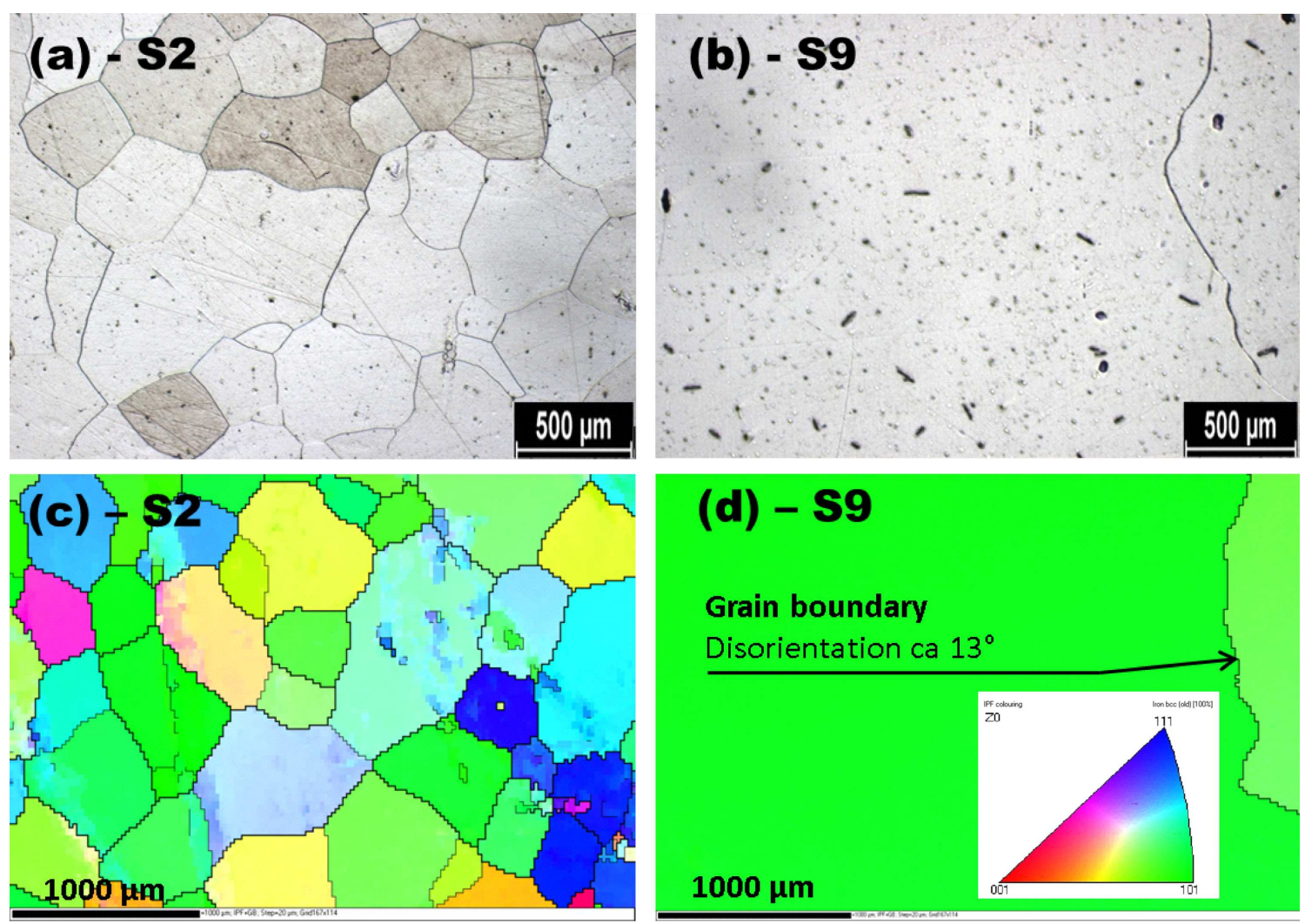

Fig. 1. Microstructure of the samples S2 and S9 with the surface coating removed, shown on optical microscopy (a,b) and SEM/EBSD (c,d) micrographs. The color scale indicates the crystallographic disorientation of the grains. Contrast of the optical micrographs enhanced by etching the specimen in a dilute nital solution.

The coefficients characterizing the correlation of other measured parameters to $B_{s}$ are collected in the last column of Table II. Apparently, there is only a moderate link between the quantity $B_{s}$ measured with aid of the standard Epstein frame and the magnetic parameters obtained from magnetic BN measurements. Such finding has been already noticed in [5] and can be likely attributed to the differences in geometry of both experimental methods, as well as to the issues related to the differences in maximum strength of the applied $H$ field (cf. also the systematically lower $r$ values of the parameters when measured at the probe excitation voltage $U=2.5 \mathrm{~V})$. Concerning the RMS of BN, the correlation analysis suggests that other factors might be of prime importance for the domain wall dynamics than are those linked to the crystallographic texture order. (The latter known, in the case of GOES materials with dominating Goss texture, be inversely proportional to the average size and disorientation of grains, as demonstrated in Fig. 1 on samples S2 and S9.)

\subsection{Anisotropy of magnetic Barkhausen noise and influence of coating layer}

Observed azimuthal courses of BN RMS measured on samples before and after removal of the coating layer (Fig. 2) show more intense signal in case of the etched samples. The effect can be interpreted as caused by en- hancement of the mean magnetic domain walls speed due to the reduction of the surface stress associated with the coating layer. The observed dependences (both before and after the coating removal) do not show any correlation with the typical angular variation of magnetic losses in GOES sheets (observed e.g. in [2]) conformal with the theoretically expected azimuthal changes of magnetocrystalline energy (Fig. 3). Such finding, suggesting that the observed RMS signal of $\mathrm{BN}$ is preferentially driven by other factors than the crystallographic texture of material, must be however taken with caution due to the known sensitivity of the azimuthal course of BN RMS signal to the excitation field strength [6].

Removal of the surface layer also has a strong influence on other parameters of the cyclic magnetization process (Fig. 4). A significant increase of the relative permeability of all tested specimens after the coating layer removal is observed for both excitation voltage levels applied. This finding is conformal with the observed behavior of BN RMS signal. At the high excitation level $U=12.5 \mathrm{~V}$, also the remanence (and, with the exception of sample S9, the coercivity, too) of all samples rises after the coating layers removal. At the low excitation level however, remanence and coercivity values show rather noisy behavior with no systematic trends, documenting the experimental difficulties associated with the low field measurements. 
$\mathrm{S} 1,12.5 \mathrm{~V}$

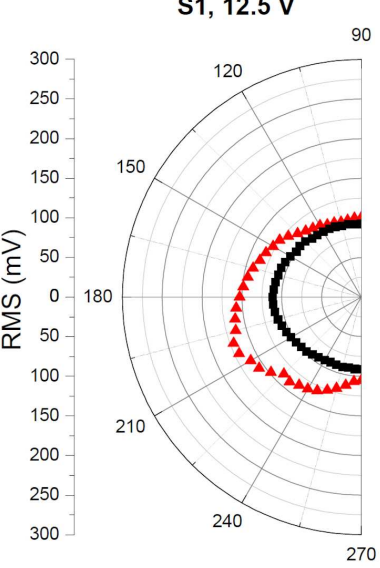

S5, $12.5 \mathrm{~V}$

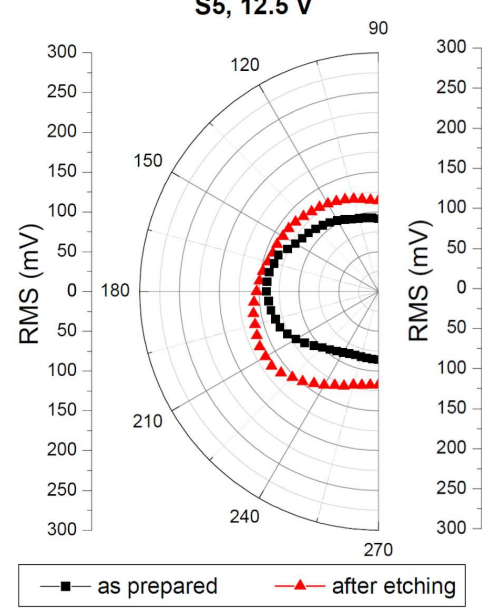

$\mathrm{S} 2,12.5 \mathrm{~V}$

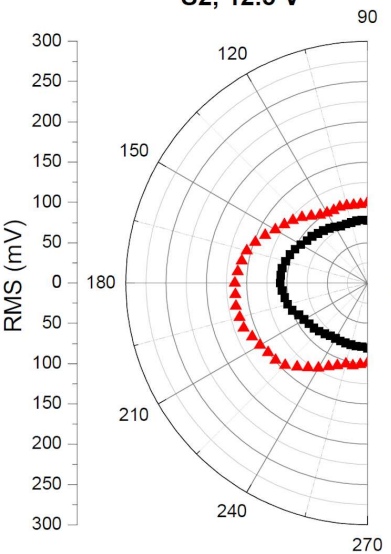

$\mathrm{S} 6,12.5 \mathrm{~V}$

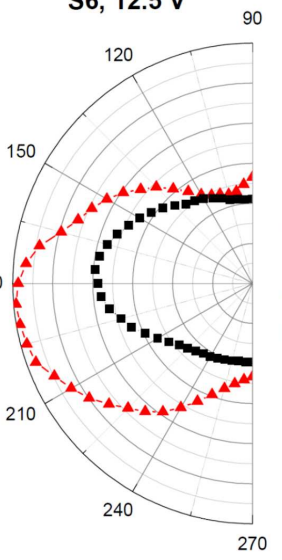

$\mathrm{S} 3,12.5 \mathrm{~V}$

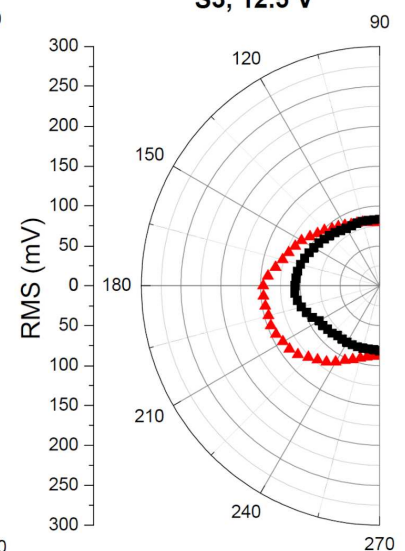

S7, $12.5 \mathrm{~V}$

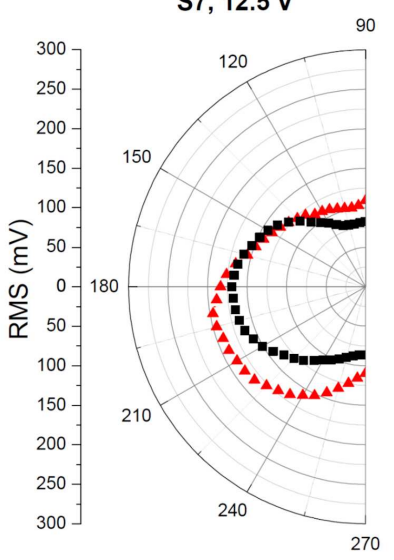

S4, $12.5 \mathrm{~V}$

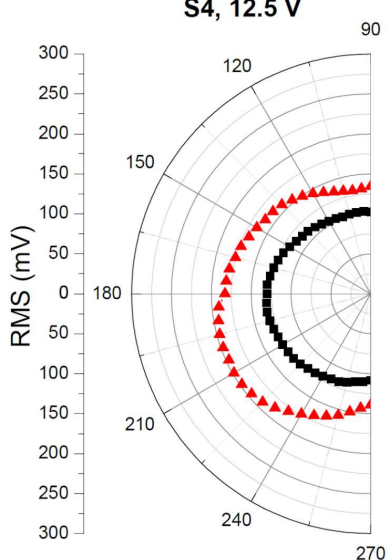

S9, $12.5 \mathrm{~V}$

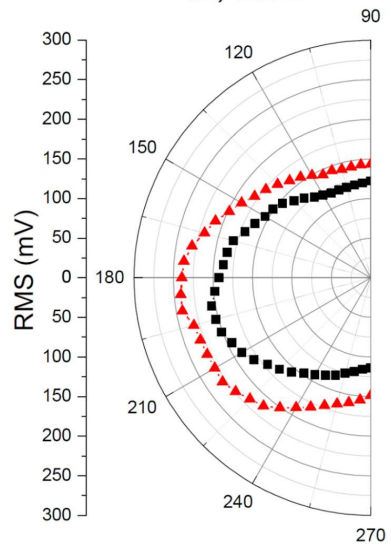

Fig. 2. The Barkhausen noise RMS signal in dependence on the azimuthal orientation of the excitation magnetic field for the samples S1-S7 and S9 before (squares) and after (triangles) removal of the coating. Excitation voltage $U=12.5 \mathrm{~V}$. The sample number is indicated.

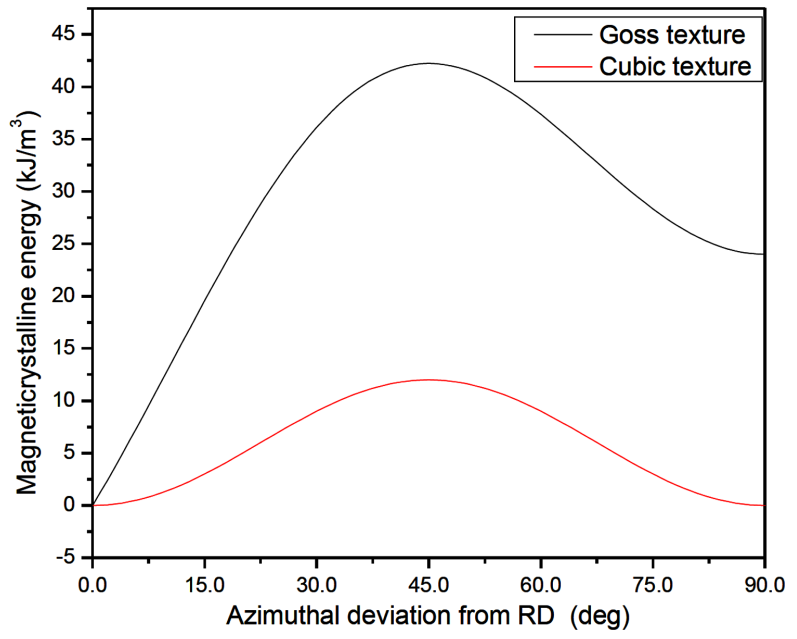

Fig. 3. Magnetocrystalline energy courses calculated for ideal Goss and cubic texture. The cubic anisotropy constants $K 1=48 \mathrm{~kJ} / \mathrm{m}^{3}$ and $K 2=50 \mathrm{~kJ} / \mathrm{m}^{3}$ overtaken from [4].

\section{Conclusions}

Crystallographic texture, microstructure and magnetic properties of the selected grain oriented electric steel samples differing in level of perfection of the Goss texture have been tested by means of neutron diffraction, scanning electron microscopy, and magnetic Barkhausen noise measurements. Pronounced correlation is found between the magnetic properties measured by standard Epstein frame technique and the texture characteristics obtained from neutron diffraction data. Only moderate correlation is observed between the texture characteristics and the results of magnetic Barkhausen noise measurements. The root mean square signal of Barkhausen noise does not follow the azimuthal profile of magnetocrystalline energy expected in case of grain oriented electric steel materials featuring a significant content of the Goss texture. Electrochemical removal of the coating layer is found to stimulate, for all the tested samples, an increase of the Barkhausen noise root mean square signal, relative permeability, remanence and coercivity. Extent of the increase varies with the intensity of the applied excitation magnetic field, being well resolved at the high field level and very noisy and uncertain at the low one. 

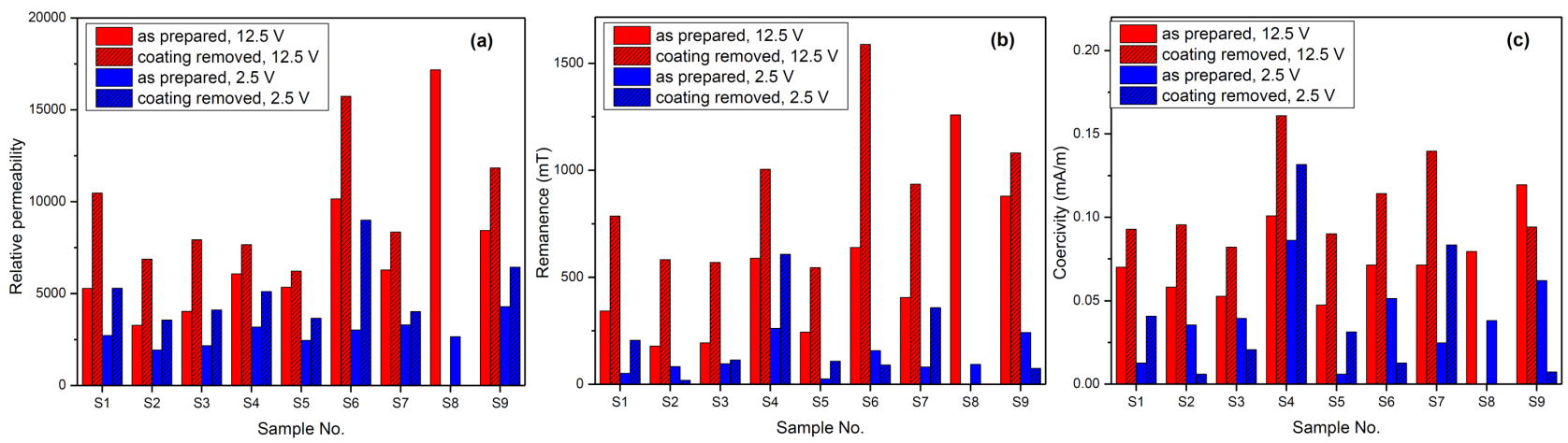

Fig. 4. Relative permeability $\mu_{r}$ (a), remanence $B_{r}(\mathrm{~b})$, and coercivity $H_{c}$ (c) measured on samples before and after coating removal with the probe excitation voltage levels $U=12.5 \mathrm{~V}$ and $2.5 \mathrm{~V}$.

\section{Acknowledgments}

The research was supported by the Czech Ministry of Education, Youth and Sports grant RVO14000, the Student grant system of the Czech Technical University in Prague grant No. SGS16/245/OHK4/3T/14, and the Czech Science Foundation grant 14-36566G.

\section{References}

[1] S.E. Zirka, Y.I. Moroz, P. Marketos, A.J. Moses, J. Magn. Magn. Mater. 320, 1039 (2008).
[2] S. Vratislav, M. Dlouhá, L. Kalvoda, Solid State Phenom. 105, 175 (2005).

[3] M. Kučeráková, K. Kolařík, J. Čapek, S. Vratislav, L. Kalvoda, J. Phys. Conf. Ser. 746, 012043 (2016).

[4] D.C. Jiles, Magnetism and Magnetic Materials, Chapman and Hall, New York 1991.

[5] O. Stupakov, O. Perevertov, V. Stoyka, R. Wood, IEEE Trans. Magn. 46, 517 (2010).

[6] P. Martínez-Ortiz, J.A. Pérez-Benítez, J.H. EspinaHernández, F. Caleyo, N. Mehboob, R. Grössinger, J.M. Hallen, J. Magn. Magn. Mater. 401, 108 (2016). 